\title{
Pseudomonas aeruginosa colonisation in an intensive therapy unit: role of cross infection and host factors
}

\author{
MALILA R NOONE, T L PITT, M BEDDER, A M HEWLETT, K B ROGERS
}

\begin{abstract}
Despite the sparsity of Pseudomonas aeruginosa in the environment colonisation and infection with this organism was found at several sites by selective culture in 20 out of 46 patients in an intensive therapy unit. Three patients developed Ps aeruginosa pneumonia. Serial serogrouping and phage typing identified multiple strains in the unit and in the same patient. Rectal carriage occurred in 16 patients but rectal strains did not subsequently appear in tracheal aspirates; strains varied in their affinity for the upper respiratory tract. Colonisation was not directly related to length of stay and was detected in 16 of those colonised within 24 hours of admission. In intubated patients, who were colonised more frequently than those not intubated, upper respiratory tract colonisation correlated strongly with low initial arterial pH values. Personnel were probably responsible for cross infection among patients when the unit was busy. Strain differences and the susceptibility of patients also influenced colonisation and infection.

Elimination of major reservoirs of Ps aeruginosa and compliance with procedures to control cross infection remain essential if patients in hospital are to escape colonisation by the organism.
\end{abstract}

\footnotetext{
Division of Communicable Diseases, Clinical Research Centre, Harrow, Middlesex

MALILA R NOONE, MB, MRCPATH, research microbiologist

Cross-infection Reference Laboratory, Central Public Health Laboratory, London NW9 5HT

T L PITT, PHD, senior microbiologist

Northwick Park Hospital, Harrow, Middlesex

M BEDDER, visiting student

A M HEWLETT, MB, FFARCS, consultant anaesthetist and chairman of intensive therapy unit

K B ROGERS, MD, FRCPATH, consultant microbiologist
}

\section{Introduction}

Patients in intensive therapy units are especially susceptible to colonisation and infection with Pseudomonas aeruginosa ${ }^{12}$; those who develop pneumonia have a high mortality even when treated with apparently effective antibiotics. ${ }^{3}$ Ps aeruginosa is commonly found in the hospital environment; several reservoirs of infection have been identified and linked with outbreaks of infection. ${ }^{4-6}$ Little is known, however, about the relative importance of endogenous sources, the prevalence of strain differences, or the influence of host factors on colonisation and infection. We have therefore conducted a prospective study to investigate these aspects in patients admitted to the intensive therapy unit of a district general hospital in which steps had already been taken to eliminate environmental reservoirs.

\section{Patients and methods \\ ACCOMMODATION AND EQUIPMENT}

In this hospital the intensive therapy unit is sited away from all wards, is adjacent to the recovery room of the surgical suite, and has two adjoining open cubicles and two isolation rooms opening on to a common corridor. The open cubicles, which were most frequently used, each contained two beds with a floor area of $12 \mathrm{~m}^{2} / \mathrm{bed}$. Washbasins with elbow taps were available in each cubicle and isolation room and two more were situated in the corridor. The waste traps of the two washbasins in the open cubicles were heated twice daily. Plenum ventilation provided eight air changes each hour. Siliconised Williams filters were fitted in both the inspiratory and expiratory circuits of the Brompton Manley ventilators and autoclaved after use. The 900B Servo ventilator (Siemens) used for children had autoclavable gas conveying parts. Heat-moisture exchange units (Servo $150 / 151$ ) were used for all ventilated patients and changed daily. Humidifiers for East-Radcliffe blowers contained aqueous chlorhexidine $0.3 \%$ heated to $55^{\circ} \mathrm{C}$. Other equipment was either autoclaved daily (ventilatory tubing, suction apparatus) or disposable (tracheal suction catheters, endotracheal tubes, nebuliser units).

Patients recovering from operations and those with severe trauma who required intensive nursing care, and all patients with acute respiratory failure were admitted to the unit. Each patient was cared for by one nurse, though occasionally nurses helped with other patients. Freshly laundered uniforms (trousers and short sleeved tunic top) were put on at the beginning of each eight hour period of duty. To protect 
the patient disposable gloves and plastic aprons were worn during sterile procedures such as tracheal suction. Hibiscrub $(4 \%$ chlorhexidine in detergent) was used for handwashing. When nurses considered that their hands were clean they applied Hibisol (chlorhexidine in alcohol) to them immediately before handling a patient.

\section{BACTERIOLOGICAL SAMPLING}

Patients-Specimens were obtained before or as soon as possible after admission to the unit and then once daily throughout the patient's stay. Samples were also collected from intubated patients every eight hours for the first 24 hours. Skin sites (external ear, groin, fourth toe web) were sampled using swabs moistened with nutrient broth. Swabs of the oropharynx and rectum, tracheal aspirate, and urine were cultured; wounds and intravenous catheter tips were cultured when indicated.

Environment-Ventilators, suction apparatus, humidifier water, patient monitoring equipment, sinks, taps, utensils used in patient care, specimens of food, detergent solutions, and mouthwash were sampled using moistened swabs for dry surfaces.

Nurses and physiotherapists-Hands were sampled using a glove wash technique with $50 \mathrm{ml} 0 \cdot 1 \%$ Triton $\mathrm{X}-100$ in $0 \cdot 1 M$ phosphate buffer $(\mathrm{pH} 7 \cdot 9) .{ }^{8}$ When gloves were worn during a procedure the used gloves were inverted and sampled using the same solution; hands were sampled by contact impression on culture plates. Pourplate cultures were made from $20 \mathrm{ml}$ of the sampling fluid. Nurses' forearms were swabbed and the lower front of their uniforms sampled using RODAC contact plates.

\section{CULTURE AND TYPING}

Surveillance samples other than those from hands and uniforms were transported in nutrient broth with $0.03 \%$ cetrimide and enriched for 24-36 hours at $37^{\circ} \mathrm{C}$ before subculture. King's B medium containing $0.03 \%$ cetrimide was used for the culture of all specimens. Cultures producing fluorescin within 48 hours at $37^{\circ} \mathrm{C}$ were confirmed as $P$ s aeruginosa if they produced pyocyanin on King's A medium or grew at $42^{\circ} \mathrm{C}$. For comparison the first 155 specimens from patients were cultured within eight hours of collection as well as after enrichment. All isolates of Ps aeruginosa were serotyped and phage typed at the Central Public Health Laboratory, London. Strains of the same O serogroup were considered distinct only if three or more strong reaction differences occurred in the phage typing pattern. ${ }^{1011}$

\section{CLINICAL DATA AND NEUTROPHIL FUNCTION}

Patients were examined daily and clinical data recorded on vital signs, evidence of infection, presence of artificial airways, mechanical ventilation, biochemical and haematological variables, and treatment with antimicrobial and other agents. Pneumonia was defined as "definite" by the criteria of Johanson et al ${ }^{12}$ - that is, fever, leucocytosis, purulent tracheobronchial secretions, and the appearance of new or progressive pulmonary infiltrates on $x$-ray films. If one of the last two features was absent pneumonia was defined as "probable."

A standard neutrophil bactericidal assay ${ }^{13}$ was performed in vitro on separated neutrophils from all patients with $P s$ aeruginosa pneumonia within three days of the onset of infection. The patient's own cells and serum were tested in duplicate against his own tracheal isolate with appropriate controls. Results were expressed as percentage reduction in colony forming units from the original bacterial challenge.

\section{STATISTICAL ANALYSIS}

Results were analysed by the Mann-Whitney rank sum test and $\chi^{2}$ tests (Minitab: Pennsylvania State University Computation Center).

\section{Results}

\section{COLONISATION}

Of 63 patients admitted over 13 weeks, 48 were screened for carriage of Ps aeruginosa. The 15 patients who were not screened had not been expected to stay long and had a mean length of stay of 7.4 hours. Two patients who were admitted from other hospitals were already intubated and heavily colonised with Ps aeruginosa; they are excluded from tables I and II. Of 155 patient specimens cultured within eight hours as well as after enrichment, Ps aeruginosa was isolated from 29 on primary inoculation and from a further 19 after enrichment. With enrichment culture Ps aeruginosa was detected earlier in the rectum and upper respiratory tract; without it colonisation of some skin sites would not have been detected. The results of enrichment culture are given throughout.

TABLE I-Sites of colonisation with Ps aeruginosa

\begin{tabular}{|c|c|c|c|c|c|c|c|}
\hline & \multicolumn{7}{|c|}{ No $\left({ }_{0}^{\circ}\right)$ of patients colonised with $P$ s aeruginosa: } \\
\hline & Total & Rectum & Pharynx & Trachea & Groin & Toe web & Ear \\
\hline \multirow{2}{*}{$\begin{array}{l}\text { Intubated }(n=27) \\
\text { Not intubated } \\
\quad(n=19)\end{array}$} & $15(56)$ & $12(44)$ & $7(26)$ & $6(22)$ & $9(33)$ & $5(19)$ & $4(15)$ \\
\hline & $5(26)$ & $4(21)$ & $1(5)$ & & $3(16)$ & $1(5)$ & $1(5)$ \\
\hline Total $(n=46)$ & $20(43)$ & $16(35)$ & $8(17)$ & $6(13)$ & $12(26)$ & $6(13)$ & $5(11)$ \\
\hline
\end{tabular}

Of 46 patients, 20 were colonised (table I). Of six patients sampled before elective surgery and admission to the unit, only one was colonised (rectal carriage). Different serotypes were sometimes found at different sites in the same patient. The same serotype tended to predominate at any given site, though occasionally it was replaced by another. It is possible that these sites were colonised with multiple strains but only the predominant one was detected.

Rectal carriage-The site most frequently colonised was the rectum (16 patients). In 12 patients rectal carriage was detected within eight hours of admission to the unit and in seven within 24 hours of hospital admission.

Rectal carriage and tracheal colonisation-Tracheal colonisation occurred in only five of the 12 ventilated patients with rectal carriage of Ps aeruginosa. Only one of these five patients had the same serotype at both sites, and tracheal colonisation preceded isolation from rectal swabs by two days.

Pharyngeal colonisation-Five of the six patients with tracheal colonisation also had pharyngeal colonisation but in only one pacient did pharyngeal colonisation precede tracheal colonisation. In two patients tracheal colonisation occurred first and two patients were colonised at both sites simultaneously.

Relation to length of stay-In 16 patients colonisation was evident within 24 hours of admission to the unit and in 19 by the third day. The number of sites colonised and number of strains identified increased with the duration of stay. Patients who had one or two sites colonised and in whom only one strain was identified had a mean duration of stay of three days, whereas those who had more than two

TABLE II-Clinical variables: correlation with tracheal intubation and colonisation with Ps aeruginosa

\begin{tabular}{lccccccc}
\hline & No of & \multicolumn{2}{c}{$\begin{array}{c}\text { Mean age } \\
\text { (years) }\end{array}$} & $\begin{array}{c}\text { Mean systolic } \\
\text { blood pressure } \\
\text { (mm Hg) }\end{array}$ & $\begin{array}{c}\text { Mean diastolic } \\
\text { blood pressure } \\
\text { (mm Hg) }\end{array}$ & $\begin{array}{c}\text { Mean arterial } \\
\text { pH }\end{array}$ & $\begin{array}{c}\text { Mean duration of Mean duration of } \\
\text { ventilation } \\
\text { (hours) }\end{array}$ \\
\hline stay in unit \\
(days)
\end{tabular}


sites colonised or in whom more than one strain was identified had a mean duration of stay of six days. In eight of the nine patients with upper respiratory tract colonisation this was detected within 72 hours of admission to the unit.

Influence of host factors-Patients with tracheal intubation were colonised with Ps aeruginosa more frequently than those without (table II). No significant differences between intubated and nonintubated patients were found with regard to sex distribution, age, mean initial blood pressure, peripheral white cell count, or haemoglobin concentration. The mean initial arterial $\mathrm{pH}$ value among intubated patients was, however, low $(7 \cdot 31)$ compared with the mean value among non-intubated patients in whom it was measured (7.38). Of the 27 intubated patients initial biochemical, haematological, and clinical values were compared among 12 non-colonised patients, seven colonised at sites other than the trachea and pharynx, and eight with upper respiratory tract colonisation. No significant differences were found in their age, sex, initial systolic and diastolic blood pressures, peripheral leucocyte count, haemoglobin and urea concentrations, and duration of stay in hospital before admission to the unit. The initial arterial $\mathrm{pH}$ was, however, significantly lower in patients with upper respiratory tract colonisation ( $p=0 \cdot 007$, Mann-Whitney). There were no differences in the numbers of patients receiving antibiotics before or on admission and no patients were receiving long term antibiotic treatment. Three patients were treated for pneumonia, and in these the results of neutrophil bactericidal tests were within normal limits. Results ranged from $97 \%$ to $99 \%$ reduction in colony forming units (mean $98 \%$ ). Control values ranged from $93 \%$ to $99 \%$ (mean $97 \%$ ).

\section{INFECTION}

Of the three patients treated for Ps aeruginosa pneumonia, two had definite infections and the third a probable infection. In all patients infection was apparent two to three days after initial tracheal colonisation. There were no urinary tract or soft tissue infections with Ps aeruginosa.

Cross infection-Ten serotypes of Ps aeruginosa were identified among isolates from 22 patients including the two already colonised on admission. Phage typing distinguished 16 strains. Six patients whose periods of admission overlapped had upper respiratory tract colonisation with strains of serogroup O11 showing the phage pattern 44,119 , 1214 (designated 11A). This strain was first isolated from a patient admitted with a heavily colonised tracheostomy. During the first four weeks of his stay no isolations of $11 \mathrm{~A}$ were obtained from any other patient. Over the succeeding two weeks-that is, weeks 5 and 6 of the study-this strain was isolated several times from five ventilated
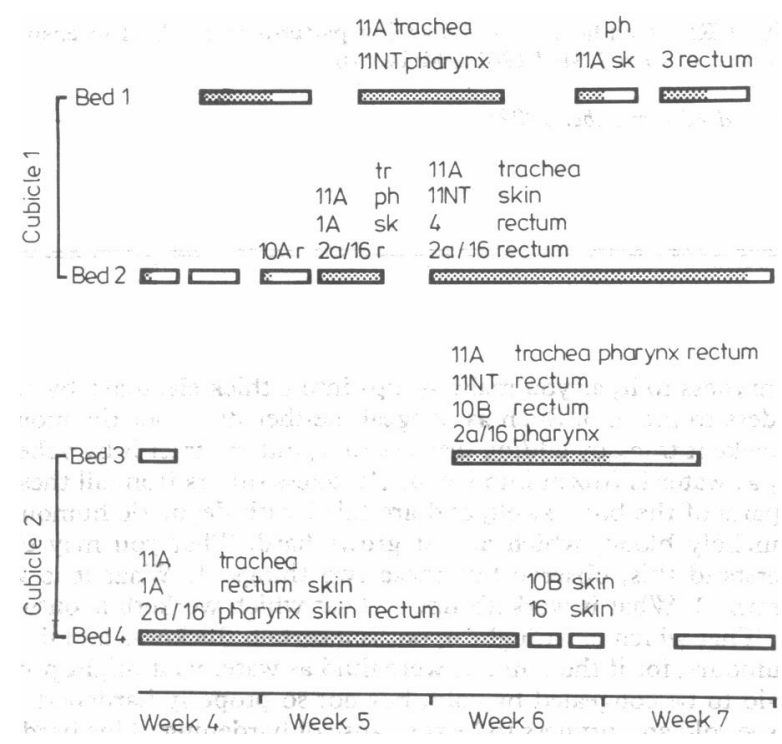

Strains of Ps aeruginosa isolated from patients during weeks 4-7 of study. Occupancy of each of four beds shown on different line on which each rectangle refers to stay of individual patient. Tracheal intubation indicated by shading of rectangle. Ps aeruginosa serogroups denoted by numerals and distinct phage types by letters A and B. NT=Non-typable. The main source of each strain also indicated: $\mathrm{tr}=$ trachea; $\mathrm{ph}=$ pharynx; $\mathrm{r}=$ rectum; sk $=$ skin. patients, three of whom developed pneumonia (figure). This coincided with a pronounced increase in work load in the unit. Though six other distinguishable strains were isolated from these six patients only strain $11 \mathrm{~A}$ showed epidemic spread. In five other instances two patients whose stay in the unit overlapped carried indistinguishable strains of Ps aeruginosa; but in 13 instances isolates from patients did not correspond to those from other patients or the environment. Nontypable strains were not compared.

Environment-The only sites from which Ps aeruginosa was isolated were washbasin waste traps and two cold water taps. Only seven of 48 samples from heated waste traps grew Ps aeruginosa compared with three quarters of those from unheated ones. The epidemic strain $11 \mathrm{~A}$ was identified among isolates from waste traps but not immediately before patient colonisation. Strains isolated from swabs of two cold water taps could not be recovered from $50 \mathrm{ml}$ tap water and could not be traced to any patients.

Nurses and physiotherapists-Right and left hands were sampled together on 37 occasions. Right hands were contaminated on 14 occasions but left hands on only four. Hand contamination occurred most often after tracheal toilet on already colonised patients, the corresponding glove being heavily contaminated. The serotype identified corresponded with the strain in the patient's trachea and pharynx. No samples were positive after washing or after handling a non-colonised patient. Forearm swabs were rarely positive (one out of 30 samples). Eleven of 36 uniform samples grew Ps aeruginosa. These were taken after varying periods of wear up to six hours. Serotypes identified from uniforms did not always correspond with the single patient allocated to each nurse.

\section{Discussion}

This study showed colonisation with Ps aeruginosa in 20 out of 46 patients, some of whom would not have been identified by routine microbiological surveillance. The pattern of colonisation was sometimes complex; the predominant strain changed at individual sites, and multiple strains were found after prolonged stay in the unit. Since samples were selectively cultured for $P s$ aeruginosa the influence, if any, of other organisms could not be assessed.

The rectum was the most frequently colonised site (16 cases). In seven patients rectal colonisation was probably acquired before admission to hospital, as it was detected within 24 hours of admission; a further five were colonised by the time they were admitted to the unit. Some aspect of illness or stay in hospital may possibly unmask colonising strains which would not otherwise be detectable; this may account for the high incidence of early rectal colonisation. Despite its frequency, however, we could not find a causal link between intestinal colonisation and respiratory tract colonisation and infection; strains isolated from the rectum were not the same as those subsequently found in the trachea. Tracheal colonisation appeared to be acquired directly rather than endogenously. Pharyngeal strains were usually similar to tracheal strains, but pharyngeal colonisation seldom occurred first.

Not all strains showed the same ability to colonise the trachea and pharynx. A strain designated $11 \mathrm{~A}$ colonised the upper respiratory tract of six patients causing pneumonia in three, though several other strains were isolated from other sites and the environment at the same time. Though several toxic products of Ps aeruginosa have been identified, ${ }^{14}$ no significant correlation between strain types and pathogenicity has yet been established. Further investigation of these bacterial products and of properties such as adherence may explain the apparent affinity of particular strains for the upper respiratory tract.

Since patients who were intubated were colonised more frequently than those who were not interference with local defences might have been a factor. Upper respiratory tract colonisation occurred rapidly, however, and was not directly related to the duration of intubation or mechanical ventilation. Johanson $e t a^{15}$ also noted rapid colonisation of the pharynx by Gram negative bacilli in patients admitted to hospital and suggested that those who became colonised were uniquely susceptible; colonisation correlated with the clinical severity of the illness rather than with the use of antibiotics or length of stay 
in hospital. The exact mechanism and relation to stress is obscure, but in our study, in which all patients were critically ill, colonisation of the trachea and pharynx correlated with low arterial $\mathrm{pH}$ values on admission. This merits further examination, as acidosis interferes with pulmonary clearing of $P s$ aeruginosa in rats ${ }^{16}$ and with macrophage function. Neutrophil bactericidal function was within normal limits in three patients who were investigated after developing pneumonia. Testing for antibacterial activity in cells and serum when the patient is acutely ill would be more appropriate.

The general pattern of strain distribution in the intensive therapy unit was a common one, in which unrelated types appeared sporadically with an episode of cross infection with a single strain designated 11A. This strain could not be traced to an environmental source; reservoirs of $P$ s aeruginosa in the environment had been reduced to a minimum. That there were no infections associated with contaminated ventilators or urinary catheters, which are the most frequent sources of Ps aeruginosa infection, is noteworthy. As in other studies, strains in sink waste traps did not cause infection. Since hands can be contaminated by splashback, ${ }^{7}$ unheated waste traps may, however, be a hazard when heavily contaminated with strains from infected patients or when standards of hygiene slip because of pressure of work.

Hands were contaminated after handling of a colonised patient, especially after tracheal aspiration, even though gloves were worn. Hands were clean when sampled at other times and after washing, but this may not truly reflect what happens in practice. In another study poor compliance with recommended handwashing practices by unit staff occurred even under normal working conditions. ${ }^{17}$ Since standards of hygiene are more likely to fall when the unit is busy the patients with tracheal colonisation may have been cross infected with strain $11 \mathrm{~A}$. Interestingly, before this episode the index patient carrying $11 \mathrm{~A}$ in the trachea was nursed in the unit for four weeks without any evidence of cross infection. Since colonisation may not be detected, or may be detected late by routine microbiological methods, some patients may not be recognised as sources of infection. This could be particularly dangerous in multibedded open cubicles, which facilitate nursing care but in which a strict code of barrier nursing is more difficult to maintain.

The elimination of major reservoirs and the control of cross infection continue to be essential in the control of Ps aeruginosa infection. Some patients are apparently more easily colonised than others; acidosis appears to be a host factor predisposing to upper respiratory tract colonisation. The development of serious infection among those colonised may also depend on the bacterial strain.
We thank Latika Shah and G Reynolds for technical work and Dr D A J Tyrrell and Dr Elliott Larson for considerable help and advice throughout the study.

\section{References}

${ }^{1}$ Bryant LR, Trinkle JK, Mobin-Uddin K, Baker J, Griffen WO Jr. Bacterial colonisation profile with tracheal intubation and mechanical ventilation. Arch Surg 1972;104:647-51.

${ }^{2}$ Stevens RM, Teres D, Skillman JJ, Finegold DS. Pneumonia in an intensive care unit: a 30-month experience. Arch Intern Med 1974;134: 106-11.

${ }^{3}$ Noone P, Abeysundere RL, Amirak I, Bradley JM, Chipping P, Perera M. Experience with aminoglycosides in life-threatening infections. Proceedings of 10th congress of chemotherapy. Current Chemotherapy 1978; 2:1016-8.

4 Phillips I, Spencer G. Pseudomonas aeruginosa cross-infection due to contaminated respiratory apparatus. Lancet 1965;ii:1325-7.

5 Ayliffe GAJ, Lowbury EJL, Hamilton JG, Small JM, Asheshov EA, Parker MT. Hospital infection with Pseudomonas aeruginosai n neurosurgery. Lancet 1965 ;ii:365-9.

${ }^{6}$ Lowbury EJL, Thom BT, Lilly HA, Babb JR, Whittal K. Sources of infection with Pseudomonas aeruginosa in patients with tracheostomy. f Med Microbiol 1970;3:39-56.

${ }^{7}$ Kohn J. A waste-trap sterilising method. Lancet 1970;ii:550-1.

${ }^{8}$ Michaud RN, McGrath MB, Goss WA. Improved experimental model for measuring skin degerming activity on the human hand. Antimicrob Agents Chemother 1972;2:8-15.

9 Brown VI, Lowbury EJL. Use of an improved cetrimide agar medium and other culture methods for Pseudomonas aeruginosa. $f$ Clin Pathol 1965; 18:752-6.

10 Asheshov EH. An assessment of the methods used for typing strains of Pseudomonas aeruginosa. In: Arseni A, ed. Proceedings of sixth national congress of bacteriology. Athens: Leontiadi Medical Editions, 1974

11 Pitt TL. A comparison of flagella typing and phage typing as a means of subdividing the $\mathrm{O}$ groups of Pseudomonas aeruginosa. $\mathcal{F}$ Med Microbiol 1981;14:261-70.

12 Johanson WG, Pierce AK, Sanford JP, Thomas Grace D. Nosocomial respiratory infections with Gram-negative bacilli. The significance of colonisation of the respiratory tract. Ann Intern Med 1972;77:701-6 .

13 Quie P, White JG, Holmes B, Good RA. In-vitro bactericidal capacity of human polymorphonuclear leukocytes: diminished activity in chronic granulomatory disease of childhood. $\mathcal{F}$ Clin Invest 1967;46:668-79.

${ }^{14}$ Liu PV. Toxins of Pseudomonas aeruginosa. In: Doggett RG, ed. Pseudomonas aeruginos a clinical manifestations of infection and current therapy New York: Academic Press, 1979.

15 Johanson WG, Pierce AK, Sanford JP. Changing pharyngeal bacterial flora of hospitalised patients: emergence of Gram-negative bacilli. $N$ Engl f Med 1969;281:1137-40.

16 Goldstein E, Green GM, Seamans C. The effect of acidosis on pulmonary bactericidal function. F Lab Clin Med 1970;75:912-23.

17 Albert RK, Condie F. Hand-washing patterns in medical intensive care units. $N$ Engl F Med 1981 ;304:1465-6.

(Accepted 26 November 1982)
HARDENING MEDICINES-Galen in Lib. 5. de Simple, Med. Facult. Cap. 10. determines hardening medicines to be cold and moist, and he brings some arguments to prove it, against which other physicians contest. I shall not here stand to quote the dispute, only take notice, that if softening medicines be hot and moist (as we shewed even now) then hardening medicines must needs be cold and dry, because they are contrary to them. The universal course of nature will prove it, for dryness and moisture are passive qualities, neither can extremities consist in moisture as you may know, if you do but consider that dryness is not attributed to the air, nor water, but to the fire, and earth. 2. The thing to be congealed must needs be moist, therefore the medicine congealing must of necessity be dry, for if cold be joined with dryness, it contracts the pores, that so the humours cannot be scattered.

Yet you must observe a difference between medicines drying, making thick, hardening, and congealing, of which differences, a few words will not do amiss. 1 . Such medicines are said to dry, which draw out, or drink up the moisture, as a spunge drinks up water. 2. Such medicines are said to make thick, as do not consume the moisture, but add dryness to it, as you make syrups into a thick electuary by adding powders to them. 3. Such as congeal, neither draw out the moisture, nor make it thick by adding dryness to it, but contract it by vehement cold, as water is frozen into ice. 4. Hardness differs from all these, for the parts of the body swell, and are filled with flegmatic humours, or melancholy blood, which at last grows hard. That you may clearly understand this, observe but these two things. 1. What it is which worketh. 2. What it worketh upon. That which worketh is outwardly cold. That which is wrought upon, is a certain thickness and dryness, of humours, for if the humour were fluid as water is, it might properly be said to be congealed by cold, but not so properly hardened. Thus you see cold and dryness to be the cause of hardening. This hardening being so far from being useful, that it is obnoxious to the body of man. I pass it without more words. I suppose when Galen wrote of hardening medicines, he intended such as make thick, and therefore amongst them he reckons up Fleawort, Purslain, Houseleek, and the like, which assuage the heat of the humours in swellings, and stops subtil and sharp defluxions upon the lungs. (Nicholas Culpeper (1616-54) The Complete Herbal, 1850.) 